\title{
Use of participant focus groups to identify barriers and facilitators to worksite exercise therapy adherence in randomized controlled trials involving firefighters
}

\author{
This article was published in the following Dove Press journal: \\ Patient Preference and Adherence \\ 12 March 2013 \\ Number of times this article has been viewed
}

\section{John M Mayer' \\ James L Nuzzo' \\ Simon Dagenais ${ }^{2}$}

'School of Physical Therapy and Rehabilitation Sciences, College of Medicine, University of South Florida, Tampa, FL, ${ }^{2}$ Palladian Health, West Seneca, NY, USA
Correspondence: John M Mayer

School of Physical Therapy and Rehabilitation Sciences, College of Medicine, University of South Florida, I290I Bruce B Downs Blvd, MDC77, Tampa, FL, 33612, USA

Tel +l 8I397438I8

Email jmayer2@health.usf.edu
Background: Firefighters are at increased risk for back injuries, which may be mitigated through exercise therapy to increase trunk muscle endurance. However, long-term adherence to exercise therapy is generally poor, limiting its potential benefits. Focus groups can be used to identify key barriers and facilitators to exercise adherence among study participants.

Objective: To explore barriers and facilitators to worksite exercise therapy adherence among firefighters to inform future randomized controlled trials (RCTs).

Methods: Participants enrolled in a previous RCT requiring twice-weekly worksite exercise therapy for 24 weeks were asked to take part in moderated focus group discussions centered on eight open-ended questions related to exercise adherence. Responses were analyzed qualitatively using a social ecological framework to identify key intrapersonal, interpersonal, and institutional barriers and potential facilitators to exercise adherence.

Results: A total of 27 participants were included in the four focus group discussions, representing $50 \%$ of those assigned to a worksite exercise therapy group in the previous RCT, in which only $67 \%$ of scheduled exercise therapy sessions were completed. Lack of self-motivation was cited as the key intrapersonal barrier to adherence, while lack of peer support was the key interpersonal barrier reported, and lack of time to exercise during work shifts was the key institutional barrier identified.

Conclusion: Focus group discussions identified both key barriers and potential facilitators to increase worksite exercise therapy adherence among firefighters. Future studies should consider educating and reminding participants about the benefits of exercise, providing individual and group incentives based on exercise adherence and performance, providing outside monitoring of exercise adherence, varying the exercise routine, encouraging group exercise and competition, and scheduling exercise during each work shift.

Keywords: exercise therapy, back injuries, self-motivation, peer support

\section{Introduction}

Firefighters are required to perform a variety of rigorous physical activities, including responding to medical emergencies, motor vehicle and other accidents, lifting and carrying patients, as well as fighting fires in hazardous, varied, and stressful environments. ${ }^{1}$ Such occupational demands place firefighters at a higher risk for developing musculoskeletal strains and injuries, including low back pain, which is the leading cause of early retirement from the fire service. ${ }^{2-4}$ Back and core muscles are thought to play an important role in stabilizing the spine while performing rigorous physical activities, and the level of endurance in these trunk muscles has been found 
to be predictive of the future incidence of low back pain. ${ }^{5}$ Endurance of back and core muscles may be assessed using standardized measures such as the prone static plank test and modified Biering-Sørensen test, and can be improved through supervised exercise therapy that slowly increases resistance and difficulty for targeted muscles. ${ }^{6,7}$

A recent randomized controlled trial (RCT) reported that firefighters who participated in a 24-week supervised worksite exercise therapy program (note: the term "exercise therapy" is used to denote exercise that is prescribed for a specific therapeutic purpose rather than recreational exercise) had significantly improved back and core muscular endurance at 6 months compared with those who continued to do their usual physical fitness routine. ${ }^{8,9}$ However, adherence to the twice-weekly exercise therapy protocol over 24 weeks was suboptimal, as participants completed only $67.3 \%$ (mean 32.3 ) of the 48 sessions specified in the study protocol. Further, adherence to exercise therapy sessions decreased from $69.1 \%$ of sessions in weeks $1-12$ to $65.6 \%$ in weeks 13-24, suggesting poor long-term adherence if this intervention were implemented by fire departments to reduce back injuries in firefighters.

Nevertheless, exercise therapy remains one of the most promising methods to reduce the burden of back injuries, given that more passive approaches such as ergonomic modifications, lumbar belts, and back schools have generally proven ineffective at preventing low back pain. ${ }^{10,11}$ It is therefore necessary to identify and overcome obstacles to full adherence to worksite exercise therapy in firefighters, many of whom have low levels of physical activity despite the high physical demands imposed by their occupation. ${ }^{12}$ Exploration of barriers and facilitators to exercise therapy adherence can be accomplished through qualitative analysis of moderated focus group discussions guided by open-ended questions related to those main themes. ${ }^{13}$ Similar methodology was recently used in a study that reported adherence to exercise training among firefighters was strongly affected by personal motivation, which in turn was related to the influence of colleagues and management, as well as organizational factors such as availability of time, space, and equipment to exercise. ${ }^{14}$ However, it is not currently known if similar preferences and beliefs are held by firefighters for long-term worksite exercise therapy targeting trunk muscles to improve endurance and reduce the incidence of future back injuries.

The primary objective of this study was to explore potential barriers to full adherence to worksite exercise therapy among firefighters who had recently completed a RCT involving supervised exercise therapy to improve trunk endurance. The secondary objective of this study was to suggest improvements or modifications to exercise therapy protocols to be used in future RCTs involving firefighters to minimize the impact of these barriers on adherence.

\section{Methods \\ Participants}

\section{Previous RCT}

Focus group participants were recruited from the 96 fulltime, active-duty firefighters at Tampa Fire Rescue (Tampa, FL, USA) who had enrolled in a previous RCT conducted by our research group to evaluate the effects of 24 weeks of supervised worksite exercise therapy on back and core muscle endurance. ${ }^{8}$ The RCT protocol required study participants to complete twice-weekly exercise sessions of approximately 10-15 minutes duration at their worksite and during their usual 24-hour work shifts. These sessions were scheduled ahead of time and supervised by a pool of ten peer fitness trainers (ie, firefighters with additional training in physical fitness and wellness), who often traveled from another fire station at Tampa Fire Rescue for this purpose. Participants in the RCT were eligible to receive up to US\$260 to complete the baseline and two follow-up study assessments outside of normal work hours, but were not compensated for completing the scheduled exercise therapy sessions; additional details are available elsewhere. ${ }^{8}$

\section{Focus group}

Only those 54 firefighters who were randomized to the supervised worksite exercise therapy group in the previous RCT were recruited for these focus group discussions. They were contacted by the study team by mail, telephone, and email on completing the 24 -week RCT, and offered an incentive of US\$70 to attend one of the four planned focus group discussions outside of normal work hours. All firefighters provided informed consent prior to participating in the focus group discussions, which was reviewed and approved by the University of South Florida's Institutional Review Board prior to enrollment.

\section{Procedures}

Four focus group discussions were held within a 1-week period to maximize internal consistency. Three sessions were held for participants of lower ranks (eg, firefighters), while one session was held exclusively for those of higher ranks (eg, fire captains). These groups of participants were separated to minimize the potential effects of workplace hierarchy on providing open and honest feedback. ${ }^{13}$ Each participant attended 
only one focus group, which had a target enrollment of five to ten individuals. Focus group discussions were led by a moderator (JM) and assistant moderator $(\mathrm{JN})$, who were both members of the research team that conducted the previous RCT. Participants in the focus groups were informed that the main purpose of these discussions was to provide feedback on their experience to help identify barriers and facilitators prior to implementing the worksite exercise therapy program more broadly in the fire service.

The focus group discussions were conducted using standardized methods, as described by Krueger and Casey. ${ }^{13}$ Discussions were centered on the moderator asking participants to answer eight open-ended questions intended to uncover both barriers and facilitators to adherence for worksite exercise therapy - these questions are listed in Table 1. The moderator asked each question in the order provided, and allowed sufficient time for focus group participants to provide their answers. When discussion lagged, the moderator asked additional follow-up questions intended to encourage participants to clarify and elaborate on the answers provided and elicit further discussion. Although participants were frequently encouraged by the moderator to share their thoughts, not every participant answered each question, particularly when there was apparent agreement on previous answers given by others. Each focus group discussion lasted approximately 90 minutes.

\section{Analysis}

Focus group discussions were recorded using digital recorders (DM-420 Digital Voice Recorder, Olympus Imaging America, Center Valley, PA, USA) and transcribed verbatim by an independent transcriptionist. The transcripts were then reviewed by the assistant moderator $(\mathrm{JN})$ and compared with handwritten notes taken during the focus group discussions to correct any errors and overcome periodic difficulties in the quality of the audio recording. The corrected transcripts

Table I Questions used in focus group discussions

I. What were your main reasons for participating in this study?

2. What would help motivate firefighters to participate in an exercise program?

3. What changes did you notice in your physical abilities after doing the exercises?

4. What did you like about the exercises?

5. What did you not like about the exercises?

6. What changes would you make to the exercises?

7. What would make it more practical for firefighters to perform exercises on duty?

8. What would help firefighters perform exercises over the long-term? were analyzed using a qualitative technique similar to that described by Krueger and Casey, in which individual answers to specific questions are separated from those questions and grouped into broader themes that may span several questions. ${ }^{13}$ The electronic transcripts for each question were copied and pasted into a spreadsheet (Microsoft Excel 2010, Microsoft, Redmond, WA, USA), after which verbatim answers were repeatedly shortened to uncover the main underlying themes.

This analysis was performed independently by two investigators ( $\mathrm{JN}$ and $\mathrm{SD}$ ), who then discussed their interpretation until consensus was reached. A social ecological framework was used for this analysis, which views desired health behaviors such as adherence to worksite exercise therapy as influenced by a variety of intrapersonal (eg, knowledge, beliefs), interpersonal (eg, colleague behaviors and attitudes), institutional (eg, goals, policies), community (eg, networks of institutions), and public policy (eg, laws, regulations) factors. ${ }^{14,15}$ This framework has previously been used to identify barriers and facilitators to general exercise in firefighters. ${ }^{14}$ This analysis focused on key barriers and facilitators related to intrapersonal, interpersonal, and institutional factors, which may be more amenable to modification by investigators in future studies involving exercise therapy.

\section{Results \\ Participants}

Firefighters who were assigned to the exercise therapy group in the previous RCT had an age of $37.6 \pm 9.8$ (mean \pm standard deviation [SD]), had worked $11.3 \pm 9.3$ years as a firefighter, exercised $3.2 \pm 1.2$ days/week, and had a body mass index of $28.2 \pm 3.3$; $96.3 \%$ were male; no significant differences were found between those who did and did not participate in the focus group discussion in terms of age, years as a firefighter, exercise frequency, body mass index, or sex. ${ }^{8}$ A total of 27 firefighters from the exercise therapy group in the previous RCT participated in focus group discussions, representing $50 \%$ of previous study participants.

\section{Adherence to exercise therapy}

In the previous RCT of exercise therapy for back and core muscle endurance, participants were required to complete twice-weekly sessions for 24 weeks, a potential total of 2592 exercise therapy sessions. However, only 1745 study exercise sessions occurred, resulting in an overall adherence of $67.3 \%$. Reasons reported by participants for missing scheduled exercise therapy sessions were noted by study personnel and are reported in Table 2 . Nearly one-third (31.0\%) of study exercise 
Table 2 Reasons for missing study exercise therapy sessions

\begin{tabular}{lll}
\hline Reason & $\mathbf{n}$ & $\%$ \\
\hline Leave (eg, vacation, personal, sick) & 316 & 31.0 \\
Unknown & 182 & 17.9 \\
Withdrew from study & 129 & 12.7 \\
Permanent transfer to control station & 122 & 12.0 \\
Temporary transfer to control station & 103 & 10.1 \\
Rest before planned study assessment & 39 & 3.8 \\
Out on call & 33 & 3.2 \\
Could not complete exercises (health reason) & 30 & 2.9 \\
Temporary restriction in full duty status & 24 & 2.4 \\
No exercise specialist available & 12 & 1.2 \\
Could not complete exercises (other reason) & 9 & 0.9 \\
Completing mandatory training & 8 & 0.8 \\
Not in fire station & 8 & 0.8 \\
Did not meet eligibility criteria & 3 & 0.3 \\
Total & 1018 & 100 \\
\hline
\end{tabular}

Note: n: number of exercise therapy sessions.

therapy sessions were missed because participants were on leave, while no reason was given for $17.9 \%$ of missed exercise therapy sessions; these two reasons alone accounted for nearly half $(48.9 \%)$ of the incomplete adherence observed in the previous RCT. Since participants were randomized to the two study groups (exercise therapy and control) according to their usual fire station, those temporarily assigned to fire stations without the study exercise machine (ie, Roman chair) were not able to complete scheduled exercise therapy sessions.

\section{Answers to focus group questions}

The open-ended questions used to guide the focus group discussions elicited a variety of responses from participants, and the qualitative analysis of those answers uncovered a variety of main themes for each question, which are outlined in Table 3, along with sample answers related to each of those themes (note: sample answers were edited slightly for clarity as they were often part of longer sentences). Some of the main themes identified in our analysis that recurred in the responses to numerous questions included establishing a schedule for exercise, providing incentives to exercise, supervision of exercise, and the importance of camaraderie to encourage exercise. Discussions related to overcoming the key intrapersonal, interpersonal, and institutional barriers to exercise therapy adherence are summarized further on in this article and in Table 4.

\section{Overcoming the key intrapersonal barrier to exercise therapy adherence}

The key intrapersonal barrier to completing study exercise therapy sessions was a lack of self-motivation. When asked about their initial motivation to enroll in the previous
RCT, participants cited six general factors: (1) a desire to exercise, (2) interest in exercises related to low back pain, (3) the opportunity to receive exercise supervision, (4) to foster group cohesiveness and competitiveness, (5) to receive financial incentives, and (6) for perceived health and physical fitness benefits. This understanding of the reasons underpinning the motivation to exercise could be incorporated into future studies to increase adherence to exercise therapy. Suggestions to increase exercise adherence in future studies that were made by focus group participants included providing education and periodic reminders about the specific health benefits of exercise; emphasizing the link between exercise, physical fitness, and job performance; providing individual incentives for exercise adherence and performance; and periodically changing the study exercise routine.

\section{Overcoming the key interpersonal barrier to exercise therapy adherence}

The key interpersonal barrier to completing study exercise therapy sessions was a perceived lack of peer support to engage in these exercises from other crew members, other shifts within the fire station, other fire stations, fire service management, and peer fitness trainers supervising the exercise therapy sessions. Given the importance of group cohesiveness within fire stations, firefighters indicated they were much more likely to engage in study exercise therapy sessions when their colleagues also did so. Some of the underlying themes related to peer support identified in the focus group discussions included the importance of camaraderie and competitiveness to encourage exercise adherence. Suggestions to increase exercise adherence in future studies included pairing firefighters with exercise buddies to foster mutual support and accountability; providing outside monitoring, supervision, and encouragement of adherence to the exercise program; providing group incentives for adherence to exercise; creating exercise competitions within and between fire stations; clear and public endorsement of exercise by management; and encouraging management to exercise alongside other firefighters.

\section{Overcoming the key institutional barrier to exercise therapy adherence}

The key interpersonal barrier to completing study exercise therapy sessions was having insufficient time to exercise during work shifts. One of the specific reasons cited for not having time to exercise was the necessity to respond to emergency calls while exercising. Emergency calls are difficult to predict 
Table 3 Main themes and sample answers to focus group questions

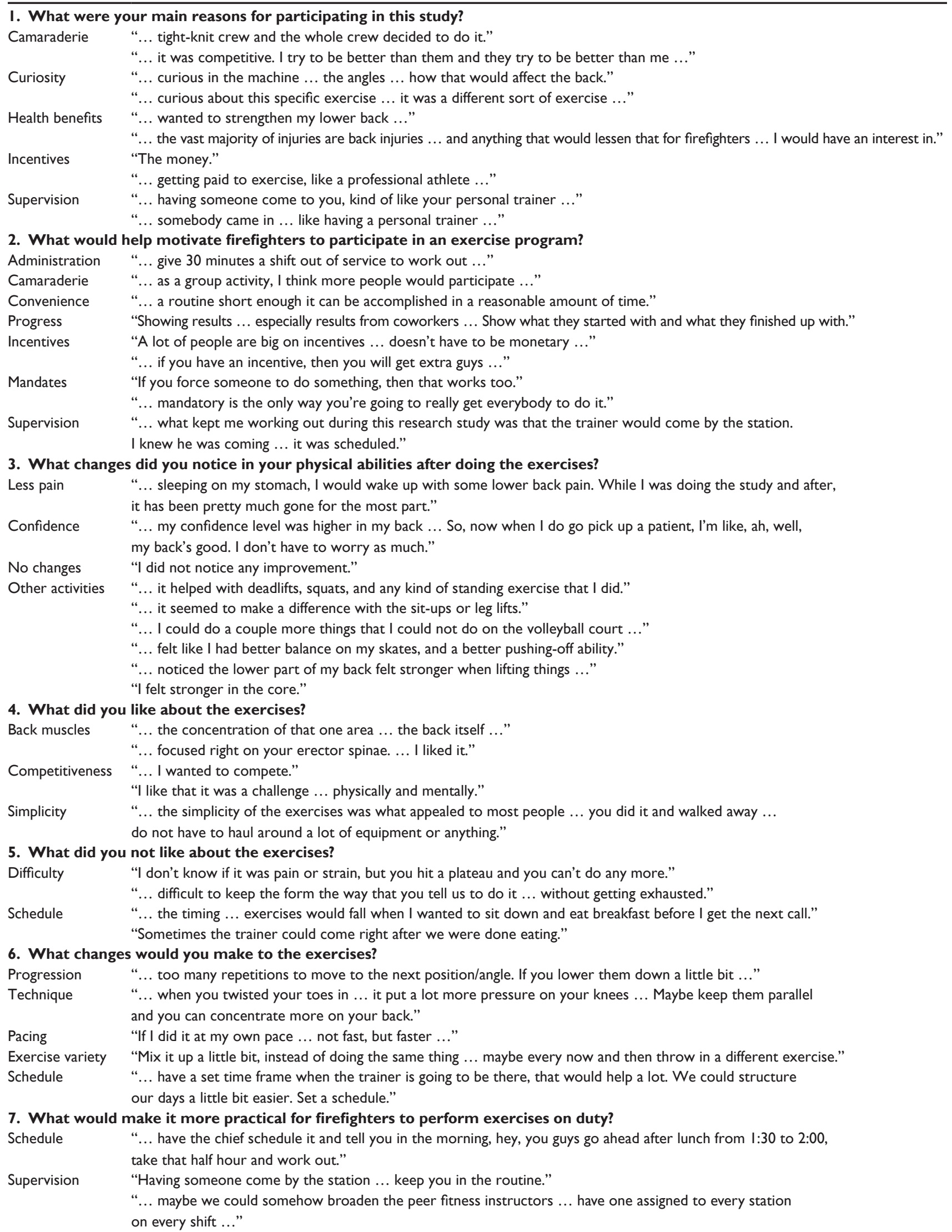


Table 3 (Continued)

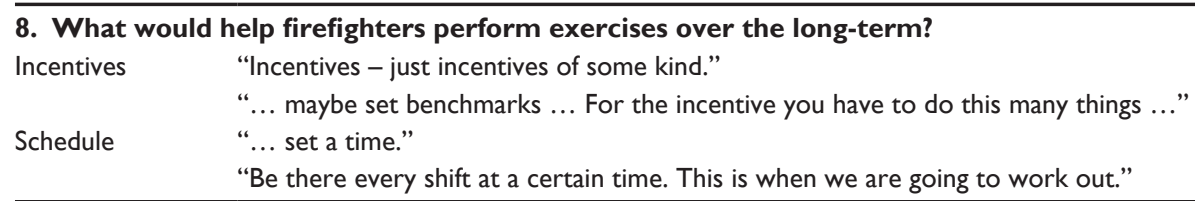

and fluctuate in frequency over time. On further discussion, focus group participants acknowledged that responding to emergency calls did not consistently prohibit adherence to study exercise therapy sessions, but occasionally interrupted them, after which it was difficult or inconvenient to resume them later due to meals, sleep, and other planned work activities. Other underlying themes identified in focus group discussions that were related to having insufficient time to exercise during work shifts included the importance of making exercises both convenient and simple to encourage adherence. Suggestions to increase exercise adherence in future studies included scheduling regular time for exercise in every shift, with scheduled back-up time slots if cancelled or interrupted; exercising as groups rather than individuals; planning meals around scheduled exercise time; taking turns covering emergency calls while others exercise; planning more frequent, shorter exercise sessions; and accepting that exercise sessions may be interrupted and resumed without harm.

\section{Discussion}

The exercise therapy protocol in the previous RCT required participants to complete twice-weekly sessions for 24 weeks to increase and maintain gains in muscular endurance in back and core muscles, which was intended to reduce their risk of future back injuries. ${ }^{8}$ However, participants completed, on average, only $67 \%$ of the desired 48 study exercise therapy sessions. Although statistically significant gains were observed in back and core endurance in study participants, this suboptimal adherence may have tempered the potential benefits of exercise therapy. ${ }^{8}$ Given that firefighters have challenging physical demands, a high prevalence of obesity, generally low levels of physical activity, and a potential increased risk of cardiovascular disease, all of which may benefit from increased adherence to exercise therapy, it is important to understand barriers and facilitators to exercise in this population. . $^{1,9,14}$

The key barriers to exercise therapy adherence identified in focus group discussions were related to lack of self-motivation, insufficient peer support to exercise, and making time to exercise during each work shift. Similar barriers have been reported in previous studies examining adherence to exercise in other settings. For example, focus group discussions were held with patients with type 2 diabetes mellitus who had previously participated in a study involving supervised exercise therapy to explore barriers and facilitators to continuing exercise adherence after the study

Table 4 Key barriers and potential facilitators to exercise therapy adherence identified in focus group discussions

\begin{tabular}{|c|c|c|}
\hline Aspect & Key barrier & Potential facilitators \\
\hline Intrapersonal & $\begin{array}{l}\text { Lack of self-motivation } \\
\text { to exercise }\end{array}$ & $\begin{array}{l}\text { - Education and periodic reminders about the specific health benefits of exercise } \\
\text { - Emphasizing the link between exercise, physical fitness, and job performance } \\
\text { - Providing individual incentives for exercise adherence and performance } \\
\text { - Periodically changing the exercise routine }\end{array}$ \\
\hline Interpersonal & $\begin{array}{l}\text { Lack of peer support } \\
\text { to exercise }\end{array}$ & $\begin{array}{l}\text { - Pairing firefighters with exercise buddies to foster mutual support and accountability } \\
\text { - Providing outside monitoring, supervision, and encouragement of adherence } \\
\text { to exercise program } \\
\text { - Providing group incentives for adherence to exercise } \\
\text { - Creating exercise competitions within and between fire stations } \\
\text { - Clear and public endorsement of exercise by management } \\
\text { - Encouraging management to exercise alongside other firefighters }\end{array}$ \\
\hline Institutional & $\begin{array}{l}\text { Lack of time to exercise } \\
\text { during work shifts }\end{array}$ & $\begin{array}{l}\text { - Scheduling regular time for exercise in every shift, with scheduled back-up } \\
\text { time slots if cancelled or interrupted } \\
\text { - Exercising as groups rather than individuals } \\
\text { - Planning meals around scheduled exercise time } \\
\text { - Taking turns covering emergency calls while others exercise } \\
\text { - Planning more frequent, shorter exercise sessions } \\
\text { - Accepting that exercise sessions may be interrupted and resumed without harm }\end{array}$ \\
\hline
\end{tabular}


was completed. ${ }^{16}$ The main themes identified were that it was challenging to sustain motivation to exercise despite knowing the health benefits of exercise, making it difficult to transition from supervised to independent exercise. Participants in that study suggested that researchers consider providing follow-up after the supervision ends to maintain the benefits achieved.

These findings are consistent with those reported in a previous study using focus group discussions to uncover barriers and facilitators to worksite physical training (ie, exercise) in firefighters. ${ }^{14}$ Motivation was the most important intrapersonal factor influencing exercise adherence, while crew dependability, cohesiveness, and group norms (ie, usual or expected beliefs and behaviors regarding exercise) were important interpersonal factors. Institutional barriers to exercise adherence included having to respond to emergency calls and other work activities, and having insufficient or inadequate space and equipment to exercise in fire stations; the required annual fitness testing was identified as an institutional facilitator to exercise, providing motivation and incentives to exercise regularly. This suggests that the barriers to worksite exercise reported by firefighters may be somewhat constant and do not vary based on the type of exercise (eg, general physical training, back endurance exercises). It is unclear if proposed facilitators for one type of exercise could also enhance participation for others.

Perceived barriers can interfere with healthy behaviors such as exercise by highlighting their disadvantages until they are perceived to outweigh their benefits, rationalizing one's decision to not engage in them. ${ }^{14,17}$ Health promotion programs must therefore anticipate and mitigate potential barriers to increase exercise participation, frequency, intensity, and duration of exercise. ${ }^{17-19}$ A study of worksite fitness center use reported that the most common barriers to exercise were insufficient time, lack of motivation, negative attitude about exercise, internal factors (eg, embarrassment), and external factors (eg, high cost) ${ }^{17}$ It also found that while reducing external barriers could increase initial exercise participation, overcoming internal barriers was necessary to enhance long-term adherence. Proposed facilitators included helping with goal-setting related to exercise adherence and performance, public recognition for achieving goals, expressing managerial support for exercise, and external monitoring of adherence. ${ }^{18,20}$

Because participating in an exercise therapy program is a voluntary decision that requires a substantial time commitment, self-motivation is necessary to initiate and sustain this effort. Since approximately $50 \%$ of those who start a new exercise program will discontinue it within 6-12 months, lack of motivation to continue exercising is a common barrier to long-term adherence. ${ }^{16,18,21,22}$ The health belief model is based on the principle that healthy behaviors are determined by health beliefs, which are in turn related to the perceived seriousness of disease(s) that can be mitigated by healthy behaviors, individual susceptibility to disease(s), the perceived benefits of engaging in healthy behavior, and potential barriers to healthy behaviors. ${ }^{20}$ To change to healthy behaviors, the perceived benefits of the new actions must outweigh their perceived costs, which can be influenced by providing financial or other incentives (ie, benefits) to help offset the time spent exercising (ie, costs). ${ }^{19,23}$

However, previous studies on financial incentives to increase exercise adherence have reported mixed findings, and some have suggested that such incentives are most commonly disbursed to those who exercise already, and are thus insufficient to entice non-exercisers to change their behavior. ${ }^{19}$ A systematic review of financial incentives for weight loss interventions - which often involve exercise therapy - reported mixed results, suggesting that such incentives achieved mostly modest, short-term effects. ${ }^{24}$ The review also noted that incentives based on changing exercise behavior (eg, increasing frequency of exercise) had a greater impact than those based solely on outcomes (eg, weight loss achieved), and that incentives for group performance were more effective than those aimed at individuals. Other factors that may influence exercise adherence include the variety of exercises, external supervision of exercise, participating in group exercise, and management support of exercise initiatives.

A study in which participants were randomized to a high-variety exercise program with ten different pieces of equipment performed $44 \%$ more repetitions, spent $18 \%$ more time exercising, and had 14\% higher levels of enjoyment than those assigned to the low-variety group that used only two pieces of equipment. ${ }^{25}$ Previous studies have also reported that the benefits of supervised exercise may be related to greater levels of external motivation, verbal encouragement, social support, increased competitiveness when performing in front of someone else, peer pressure, and faster progression through different levels of resistance when dictated by someone else. ${ }^{26,27}$ These findings are consistent with those of previous studies exploring why individuals hired personal trainers to supervise exercise, which reported that motivational skills and empathy were more appreciated than pure technical knowledge related to exercise. ${ }^{16,28}$

Firefighters often develop strong bonds with their crew members, with whom they spend extended periods, often rotating through 24-hour shifts and 48-hour periods of rest. ${ }^{3,29}$ The tight-knit structure of crews working the same 
shift encourages high levels of cohesiveness, and firefighters often schedule activities as a group, which previous studies have suggested provide a natural setting for team-based exercise programs. ${ }^{3,14}$ Facilitators to exercise adherence may include pairing firefighters with "exercise buddies" to foster mutual support and accountability. ${ }^{14}$ Since firefighters function within hierarchical organizations, management may influence healthy behaviors such as exercise, and should therefore be encouraged to exercise alongside firefighters of lower rank. ${ }^{14}$

\section{Study limitations}

There are limitations to this study that may hinder the validity and generalizability of its findings. One of the main objectives of focus group discussions is to identify important or recurring themes in a specific group of individuals that may not be otherwise apparent to those outside that group. ${ }^{14}$ This can be accomplished by having a moderator ask open-ended questions designed to elicit responses related to the themes of interest and encouraging participants to share their opinions openly. Such discussions continue until the moderator believes that a point of saturation has been reached on a given topic, after which it is unlikely that new themes will be uncovered. Findings from focus group discussions are therefore intended to be qualitative in nature, rather than tabulated and quantified as with surveys.

The qualitative analysis of transcripts from focus group discussions can potentially be influenced by the investigators' personal biases. Steps were taken to minimize this possibility by having two investigators complete their qualitative analyses independently prior to reaching consensus on important themes and key barriers.

Participation in both the previous RCT and in the focus group discussions was voluntary, making it possible that the opinions expressed were not representative of firefighters at Tampa Fire Rescue.

In addition, the barriers and potential facilitators related to adherence to worksite exercise therapy for back and core muscle endurance uncovered in focus group discussions with firefighters may not be generalizable to other study populations, settings, or forms of exercise therapy.

\section{Conclusion}

Focus group discussions were held with firefighters who had participated in a RCT involving worksite exercise therapy for back and core muscle endurance to reduce the risk of back injuries. A qualitative analysis of participant feedback uncovered key intrapersonal, interpersonal, and institutional barriers to complete exercise therapy adherence, including lack of self-motivation to exercise, lack of support to exercise from colleagues, and lack of time to exercise during work shifts. Numerous potential facilitators were suggested to improve adherence to exercise therapy in future studies. Additional research is required to determine if implementing one or more of the proposed facilitators will successfully increase adherence to exercise therapy and in turn improve the gains achieved in back and core muscle endurance to reduce back injuries. Although the barriers and facilitators identified in focus group discussions were specific to firefighters, the underlying themes may also be helpful to investigators designing exercise therapy protocols for other settings and populations.

\section{Acknowledgments}

This study was funded by the US Federal Emergency Management Agency, Department of Homeland Security (grant no EMW-2009-FP-00418). The authors wish to thank Tampa Fire Rescue for its assistance in organizing and implementing this study, as well as the focus group participants who shared their views on these topics.

\section{Disclosure}

The authors attest that they do not have any conflicts of interest, financial or otherwise, related to the subject matter discussed in this work.

\section{References}

1. International Association of Fire Fighters (IAFF). The Fire Service Joint Labor Management Wellness-Fitness Initiative. Washington DC: IAFF; 2008.

2. Karter MJ, Molis JL. US Firefighter Injuries - 2007. Quincy, MA: National Fire Protection Association; 2008. Available from: http:// www.nfpa.org/assets/files/pdf/firefighterinjuries2007.pdf. Accessed February 13, 2013.

3. Elliot DL, Goldberg L, Duncan TE, et al. The PHLAME firefighters' study: feasibility and findings. Am J Health Behav. 2004;28(1):13-23.

4. Scanlon P, Ablah E. Self-reported cardiac risks and interest in risk modification among volunteer firefighters: a survey-based study. $J \mathrm{Am}$ Osteopath Assoc. 2008;108(12):694-698.

5. Demoulin C, Vanderthommen M, Duysens C, Crielaard JM. Spinal muscle evaluation using the Sorensen test: a critical appraisal of the literature. Joint Bone Spine. 2006;73(1):43-50.

6. Biering-Sørensen F. Physical measurements as risk indicators for lowback trouble over a one-year period. Spine (Phila Pa 1976). 1984;9(2): $106-119$.

7. Verna JL, Mayer JM, Mooney V, Pierra EA, Robertson VL, Graves JE. Back extension endurance and strength: the effect of variable-angle roman chair exercise training. Spine (Phila Pa 1976). 2002;27(16): 1772-1777.

8. Mayer JM, Quillen WS. Effect of worksite exercise on development of back and core muscular endurance in firefighters. American Occupational Health Conference, Annual Meeting of the American College of Occupational and Environmental Medicine. In press 2013. 
9. Mayer JM, Nuzzo JL, Chen R, et al. The impact of obesity on back and core muscular endurance in firefighters. J Obes. 2012;729283:1-7.

10. van Poppel MN, Hooftman WE, Koes BW. An update of a systematic review of controlled clinical trials on the primary prevention of back pain at the workplace. Occup Med (Lond). 2004;54(5):345-352.

11. Burton AK, Balagué F, Cardon G, et al; COST B13 Working Group on European Guidelines for Prevention in Low Back Pain. How to prevent low back pain. Best Pract Res Clin Rheumatol. 2005;19(4): 541-555.

12. Durand G, Tsismenakis AJ, Jahnke SA, Baur DM, Christophi CA, Kales SN. Firefighters' physical activity: relation to fitness and cardiovascular disease risk. Med Sci Sports Exerc. 2011;43(9):1752-1759.

13. Krueger KA, Casey MA. Focus Groups: A Practical Guide for Applied Research, 4th ed. Thousand Oaks, CA: SAGE; 2009.

14. Staley JA, Weiner B, Linnan L. Firefighter fitness, coronary heart disease, and sudden cardiac death risk. Am J Health Behav. 2011;35(5): 603-617.

15. McLeroy KR, Bibeau D, Steckler A, Glanz K. An ecological perspective on health promotion programs. Health Educ Q. 1988;15(4):351-377.

16. Casey D, De Civita M, Dasgupta K. Understanding physical activity facilitators and barriers during and following a supervised exercise programme in Type 2 diabetes: a qualitative study. Diabet Med. 2010;27(1):79-84.

17. Schwetschenau HM, O’Brien WH, Cunningham CJ, Jex SM. Barriers to physical activity in an on-site corporate fitness center. $J$ Occup Health Psychol. 2008;13(4):371-380.

18. Shepich J, Slowiak JM, Keniston A. Do subsidization and monitoring enhance adherence to prescribed exercise? Am J Health Promot. 2007;22(1):2-5.

19. Abraham JM, Feldman R, Nyman JA, Barleen N. What factors influence participation in an exercise-focused, employer-based wellness program? Inquiry. 2011;48(3):221-241.
20. Harrison DA, Liska LZ. Promoting regular exercise in organizational fitness programs: health-related differences in motivational building blocks. Pers Psychol. 1994;41(1):47-71.

21. Andersen LL. Influence of psychosocial work environment on adherence to workplace exercise. J Occup Environ Med. 2011;53(2):182-184.

22. Webber KH, Tate DF, Ward DS, Bowling JM. Motivation and its relationship to adherence to self-monitoring and weight loss in a 16-week Internet behavioral weight loss intervention. J Nutr Educ Behav. 2010;42(3):161-167.

23. Finkelstein EA, Brown DS, Brown DR, Buchner DM. A randomized study of financial incentives to increase physical activity among sedentary older adults. Prev Med. 2008;47(2):182-187.

24. Paul-Ebhohimhen V, Avenell A. Systematic review of the use of financial incentives in treatments for obesity and overweight. Obes Rev 2008;9(4):355-367.

25. Juvancic-Heltzel JA, Glickman EL, Barkley JE. The effect of variety on physical activity: a cross-sectional study. J Strength Cond Res. 2013;27(1):244-251.

26. Mazzetti SA, Kraemer WJ, Volek JS, et al. The influence of direct supervision of resistance training on strength performance. Med Sci Sports Exerc. 2000;32(6):1175-1184.

27. Nicolaï SP, Kruidenier LM, Leffers P, Hardeman R, Hidding A, Teijink JA. Supervised exercise versus non-supervised exercise for reducing weight in obese adults. $J$ Sports Med Phys Fitness. 2009;49(1):85-90.

28. Melton DI, Katula JA, Mustian KM. The current state of personal training: an industry perspective of personal trainers in a small Southeast community. J Strength Cond Res. 2008;22(3):883-889.

29. Pillai R, Williams EA. Transformational leadership, self-efficacy, group cohesiveness, commitment, and performance. Journal of Organizational Change Management. 2004;17(2):144-159.
Patient Preference and Adherence

\section{Publish your work in this journal}

Patient Preference and Adherence is an international, peer-reviewed, open access journal focusing on the growing importance of patient preference and adherence throughout the therapeutic continuum. Patient satisfaction, acceptability, quality of life, compliance, persistence and their role in developing new therapeutic modalities and compounds to

\section{Dovepress}

optimize clinical outcomes for existing disease states are major areas of interest. This journal has been accepted for indexing on PubMed Central. The manuscript management system is completely online and includes a very quick and fair peer-review system. Visit http://www.dovepress.com/ testimonials.php to read real quotes from published authors. 